\title{
VARIACIÓN ESTACIONAL DE LA REPELENCIA DE LOS ACEITES ESENCIALES DE MONIMIACEAE SOBRE Sitophilus zeamais Motschulsky (CURCULIONIDAE)
}

\author{
SEASONAL VARIATION OF REPELLENCY OF ESSENTIAL OILS \\ OF MONIMIACEAE AGAINST Sitophilus zeamais Motschulsky \\ (CURCULIONIDAE)
}

\author{
Cristian Ortiz ${ }^{1}$, Gonzalo Silva ${ }^{1 *}$, Ernesto Moya ${ }^{1}$, Susana Fischer ${ }^{1}$, Angélica Urbina ${ }^{1}$, J. Concepción \\ Rodríguez $^{2}$ \\ ${ }^{1}$ Departamento de Producción Vegetal, Facultad de Agronomía, Universidad de Concepción, Vicente \\ Méndez 595, Chillán, Chile. \\ 2 Programa de Entomología y Acarología, Colegio de Postgraduados, Km 36,5 Carretera Federal, \\ México-Texcoco, Montecillo, Estado de México, México. \\ * Autor para correspondencia E-mail: gosilva@udec.cl
}

\section{RESUMEN}

Sitophilus zeamais (gorgojo del maíz) en cereales almacenados se controla principalmente con insecticidas sintéticos. Una alternativa son los insecticidas botánicos, especialmente los aceites esenciales. En Chile, los mejores resultados se han obtenido con los de Peumus boldus (boldo), Laurelia sempervirens (laurel) y Laureliopsis philippiana (tepa). Se estudió en laboratorio la repelencia de estos tres extractos sobre $S$. zeamais a lo largo del año. Los componentes principales de $P$. boldus fueron 1-8-cineol (14,9 a $40 \%)$ y ascaridol $(24,37 \%)$. En L. sempervirens destacaron metil-eugenol $(4,6$ a $39,7 \%)$ y safrol (38,5 a 64,7\%), y en L. philippiana safrol (17 a 39,6\%) y linalool (0,9 a 34,5\%). Salvo los extractos de L. philippiana y $P$. boldus al 0,125 y $0,25 \%$, todos los tratamientos causaron repelencia, con mayor nivel en concentraciones $\geq 4 \%$. Sólo el aceite esencial de $L$. sempervirens presentó variación estacional, con mayor repelencia en el follaje colectado en verano. La falta de efecto estacional de los aceites esenciales de $P$. boldus y $L$. philippiana se estiman promisorias para el control de plagas de granos almacenados durante todo el año.

Palabras clave: insecticidas vegetales, gorgojo del maíz, terpenos, boldo, laurel, tepa.

\section{ABSTRACT}

Sitophilus zeamais (maize weevil) in stored cereals is mainly controlled with the use of synthetic insecticides. Botanical insecticides, particularly essential oils, are an alternative to use of synthetic insecticides. In Chile, the best results have been obtained with the use of essential oils of Peumus boldus (boldo), Laurelia sempervirens (laurel) and Laureliopsis philippiana (tepa). A study was conducted in the laboratory to evaluate the repellency of these three extracts against $S$. zeamais throughout the year. The main components of each essential oil were: 1-8-cineol (from 14.9 to $40 \%$ ) and ascaridol (24.37\%) in P. boldus; methyl eugenol (from 4.6 to $39.7 \%$ ) and safrole (from 38.5 to $64.7 \%$ ) in L. sempervirens; safrol (from 17 to $39.6 \%$ ) and linalool (from 0.9 to $34.5 \%$ ) in L. philippiana. Except for the extracts of L. philippiana and P. boldus at 0.125 and $0.25 \%$, all treatments caused repellency, reaching higher levels at concentrations $\geq 4 \%$. Only the essential oil of L. sempervirens 
presented seasonal variation, with greater repellency in the foliage collected in summer. Due to the absence of seasonal effects, essential oils of $P$. boldus and L. philippiana are considered promising for the control of pests in stored grains throughout the year.

Key words: botanical insecticides, maize weevil, terpenoids, boldo, laurel, tepa.

\section{INTRODUCCIÓN}

El gorgojo del maíz, Sitophilus zeamais Motschulsky (Coleoptera; Curculionidae) es una plaga primaria mundial de cereales almacenados, que si no se controla en el almacenamiento puede destruirlos completamente en seis meses (Tefera et al., 2011). Los adultos y larvas se alimentan del endosperma; su respiración aumenta la temperatura y permite el ingreso de plagas secundarias y hongos (Trematerra et al., 2015).

Los insecticidas sintéticos son el método de control más usado contra los insectos plaga en granos almacenados. Sin embargo, S. zeamais ha desarrollado resistencia a insecticidas organofosforados (Evans, 1985; Pérez-Mendoza, 1999), piretroides (Ribeiro et al., 2003) y al fosfuro de aluminio (Pimentel et al., 2009), lo que ha incentivado la búsqueda de métodos alternativos de control, entre los que se encuentran productos naturales como los insecticidas de origen vegetal (Roel y Vendramim, 2006), siendo necesario estudiar especies chilenas que tengan esta actividad biológica (Pizarro et al., 2013).

Los mejores resultados de control se han obtenido con plantas aromáticas, en especial de la familia Monimiaceae (Bittner et al., 2008). En Chile, esta familia tiene solo tres especies nativas: Peumus boldus Molina (boldo), Laurelia sempervirens (Ruiz et Pau.) Tul (laurel) y Laureliopsis philippiana Looser (tepa) (Rodríguez et al., 2005). Bittner et al. (2008) y Betancur et al. (2010) describieron el efecto insecticida de contacto y fumigante además de actividad repelente del aceite esencial de $P$. boldus contra S. zeamais y Acanthoscelides obtectus (Say) (Bruchidae). Bittner et al. (2008) y Zapata y Smagghe (2010), encontraron que el aceite esencial de $L$. sempervirens presenta efecto insecticida fumigante sobre A. obtectus, y Tribolium castaneum (Herbst) (Tenebrionidae). En relación a la acción tóxica de L. philippiana, Ortiz et al. (2012) indicaron que el polvo de hojas secas de esta planta tiene toxicidad por contacto y causa repelencia hacia S. zeamais. A su vez, Norambuena et al. (2016) encontraron que el aceite esencial de L. philippiana, presenta un $100 \%$ de mortalidad por fumigación, un $87,9 \%$ de efecto antialimentario y todas las concentraciones evaluadas fueron repelentes.

La repelencia es una respuesta defensiva, física o química, de las plantas contra los herbívoros, directamente o a distancia, que reduce la posibilidad de una plaga para utilizarlas como alimento, refugio o sitio de ovipostura (Fancelli et al., 2005; Castro et al., 2011). En los sistemas de defensa están involucrados metabolitos secundarios (Nerio et al., 2010), los que evitan que el insecto responda al estímulo olfativo, y afectan el encuentro y reconocimiento del hospedero y por tanto causan repelencia (Rodriguez et al., 2003). Sin embargo, los insecticidas de origen vegetal presentan variabilidad en el tipo, número y concentración de compuestos químicos (Rodríguez etal., 2003), debidofundamentalmente a factores estacionales, ubicación geográfica y estado fenológico del material colectado. En esta investigación se evaluó en laboratorio la posible variación estacional de las propiedades repelente de los aceites esenciales de $P$. boldus, $L$. sempervirens y L. philippiana sobre adultos de $S$. zeamais.

\section{MATERIALES Y MÉTODOS}

La investigación se realizó en el Laboratorio de Entomología de la Facultad de Agronomía de la Universidad de Concepción, Campus Chillán, Región del Bíobío, Chile.

\section{Insectos}

Se colectaron ejemplares de $S$. zeamais de cereales infestados, en el mercado de frutas y hortalizas de Chillán, que se mantuvieron en frascos de vidrio de 1 L con maíz (Zea mays L.) Dekalb cv. DK 440 como sustrato alimenticio, a $25 \pm 1^{\circ} \mathrm{C}, 65 \pm 5 \%$ HR y $12: 12 \mathrm{~h}$ de luz:oscuridad en una cámara IPS 749 (Memmert Gmbh, Schwabach, Germany). La identificación taxonómica de los insectos se confirmó por comparación morfológica con la colección de referencia del Departamento de Zoología de la Facultad de Recursos Naturales y Oceanográficos de la Universidad de Concepción.

\section{Material vegetal}

Se colectaron hojas de P. boldus y L. sempervirens en Los Lleuques (36 $51^{\prime} 18^{\prime \prime}$ S, 71 $38^{\prime} 34^{\prime \prime}$ W, 286 msnm), Provincia de Nuble, Región del Bíobío, Chile, y L. philippiana en la Provincia de Maullín $\left(41^{\circ} 41^{\prime} \mathrm{S}, 7^{\circ} 25^{\prime} \mathrm{W}, 28 \mathrm{msnm}\right)$, Región de Los Lagos, zonas libres de polución, que en estudios previos (Pérez et al., 2007; Ortiz et al., 2012) 
presentaron resultados promisorios. Las hojas se colectaron a una altura constante y de los cuatro puntos cardinales del árbol (Vogel et al., 1997), en todas las estaciones del año, y siempre en los mismos árboles, que se identificaron con estacas. La identificación botánica se verificó por comparación anatómica con material en el herbario de la Facultad de Agronomía de la Universidad de Concepción, Campus Chillán, Chile. Estas hojas se secaron en laboratorio en un horno de convección forzada (Memmert Gmbh, UNB 500, Schwabach, Germany) a $40 \pm 5^{\circ} \mathrm{C}$ por $72 \mathrm{~h}$. El aceite esencial desde estas hojas secas se obtuvo por hidrodestilación por arrastre de vapor en un destilador Clevenger por $3 \mathrm{~h}$ (Chu et al., 2011). Los residuos de agua en el aceite esencial se eliminaron con $\mathrm{NASO}_{3}$ y los extractos se almacenaron en frascos de vidrio ámbar a $4 \pm$ $1^{\circ} \mathrm{C}$ en refrigerador.

Los aceites esenciales de las cuatro estaciones se analizaron por cromatografía de gases acoplada con espectrometría de masas GC-MS utilizando un cromatógrafo (HPGC -MS, Hewlett Packard series II 5890) de alta resolución, en el Laboratorio de Farmacognosia de la Facultad de Farmacia de la Universidad de Concepción.

\section{Repelencia}

La repelencia se evaluó siguiendo la metodología de Mazzonetto y Vendramim (2003), con modificaciones. La unidad experimental (UE) consistió en una arena de libre elección formada por cinco placas Petri plásticas de $5 \mathrm{~cm}$ de diámetro, con una placa central conectada a las otras cuatro con tubos plásticos de $10 \mathrm{~cm}$ de largo y $0,5 \mathrm{~cm}$ de diámetro, formando una $X$. En las placas periféricas se pusieron $20 \mathrm{~g}$ de maíz, impregnados sólo en placas opuestas con las concentraciones del aceite esencial a evaluar. Luego se liberaron 50 insectos adultos de $48 \mathrm{~h}$ de edad sin diferenciación de sexo en la placa central. La UE se mantuvo en cámara por 24 $\mathrm{h}$ a $25 \pm 1^{\circ} \mathrm{C}$ y se contaron los insectos en cada placa. Con estos resultados se calculó el índice de repelencia (IR) de Mazzonetto y Vendramim (2003), que clasifica el tratamiento como neutro si el IR $=1$, atrayente si el IR $>1$ y repelente si el IR $<1$. Para una calificación más precisa de los tratamientos repelentes se elaboró una escala desde sin repelencia hasta una repelencia muy alta (Tabla 1). Se evaluaron concentraciones de 0,$125 ; 0,25 ; 0,5 ; 1 ; 2 ; 4 ; y 8 \%$ de cada aceite esencial en $1 \mathrm{~mL}$ de acetona, con cuatro repeticiones y en cinco días diferentes. Además en cada repetición los tratamientos se distribuyeron al azar para evitar interferencias de factores externos como luz o temperatura.

\section{Diseño experimental y análisis estadístico}

Se utilizó un diseño experimental factorial $4 \times 7$ (cuatro estaciones del año y siete concentraciones de cada aceite esencial) completamente al azar. Los resultados se analizaron con el software InfoStat ${ }^{\circledR}$ (Di Rienzo et al., 2008), previa transformación por $X+0,5^{0,5}$ (Gómez y Gómez, 1984) para asegurar una distribución normal, para análisis de varianza del efecto de los tratamientos y pruebas de comparación de medias de Tukey $(\mathrm{P} \leq 0,05 \%)$.

\section{RESULTADOS Y DISCUSIÓN}

El componente principal del aceite esencial de $P$. boldus fue 1-8-cineol (con 35,39\% en otoño; $39,96 \%$ en invierno; $14,85 \%$ en primavera $y$ $32,3 \%$ en verano), seguido de ascaridol $(24,37 \%)$, compuesto que se encontró sólo en primavera (Tabla 2). En el aceite esencial de L. sempervirens no se detectó ascaridol, y 1-8-cineol solo se encontró en otoño y primavera en concentraciones $<2 \%$. Los compuestos en mayor concentración en el aceite esencial de L. sempervirens fueron metil-

Tabla 1. Escala complementaria al índice de repelencia de Mazzonetto y Vendramin (2003).

Table 1. Complementary scale to Mazzonetto and Vendramin (2003) repellency index.

\begin{tabular}{cl}
\hline Valor índice de repelencia (IR)* & Categoría \\
\hline$\geq 1,0$ & Sin repelencia \\
$0,76-0,99$ & Repelencia débil \\
$0,51-0,75$ & Repelencia moderada \\
$0,26-0,50$ & Repelencia alta \\
$0,0-0,25$ & Repelencia muy alta \\
\hline
\end{tabular}

\footnotetext{
* Índice de repelencia $(I R)=2 G /(G+P)$, donde $G=$ porcentaje de insectos de cada tratamiento, $y$ $\mathrm{P}=$ porcentaje de insectos en el testigo.

* Repellency index $(\mathrm{IR})=2 \mathrm{G} /(\mathrm{G}+\mathrm{P})$, where $\mathrm{G}=$ percentage of insects in each treatment, and $\mathrm{P}=$ percentage of insect in the control treatment.
} 


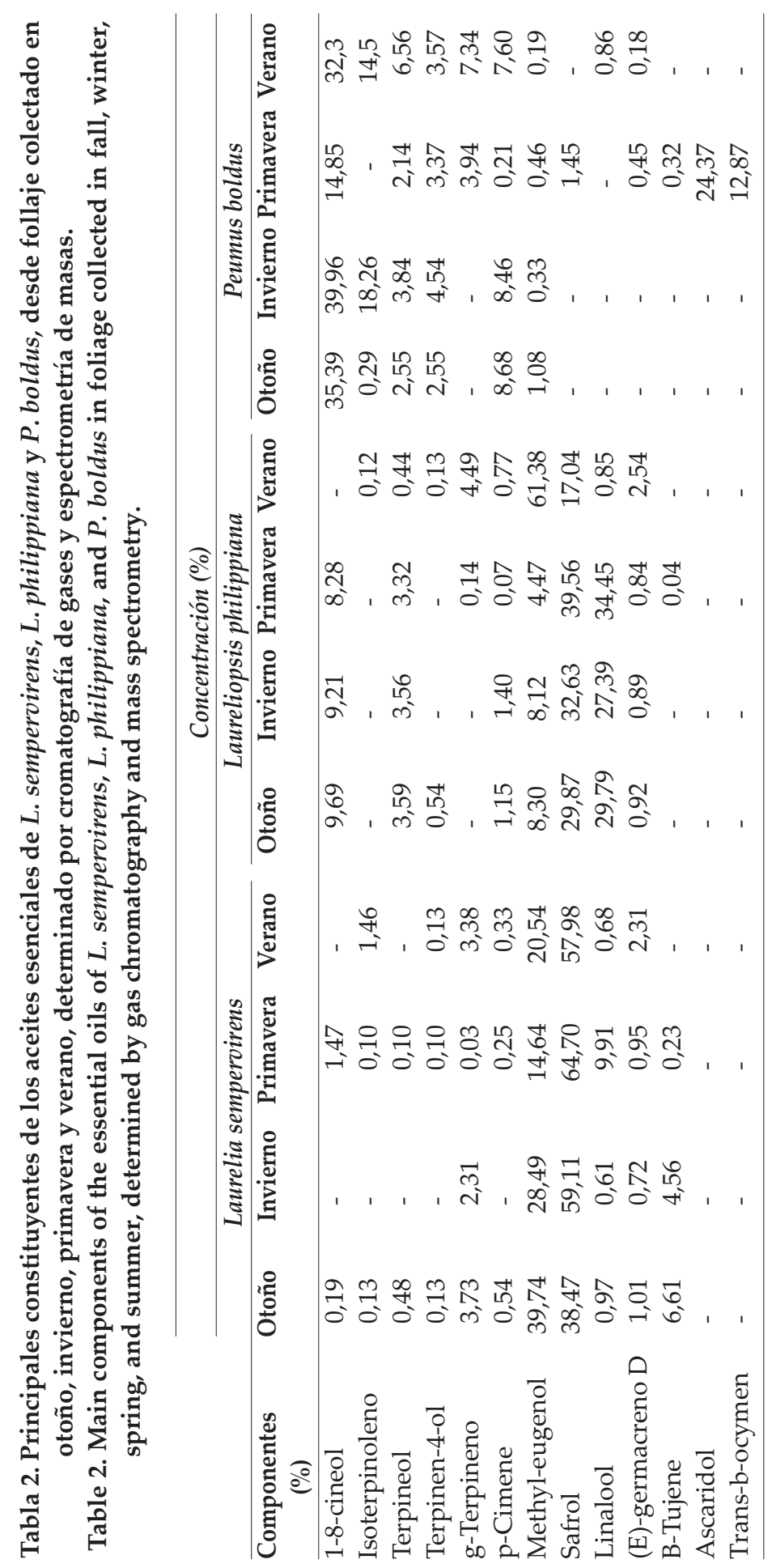


eugenol (39,74\% en otoño, $28,49 \%$ en invierno, $14,64 \%$ en primavera y $20,54 \%$ en verano) y safrol (38,47\% en otoño, $59,11 \%$ en invierno, $64,7 \%$ en primavera, y $57,98 \%$ en verano). El safrol también se encontró en concentraciones significativas en $L$. philippiana ( $29,87 \%$ en otoño, $32,63 \%$ en invierno, $39,56 \%$ en primavera, y $17,04 \%$ en verano), $y$ linalool en alta proporción $(29,79 \%$ en otoño, $27,39 \%$ en invierno, $34,45 \%$ en primavera y $0,85 \%$ en verano) (Tabla 2).

En los tres aceites esenciales evaluados, la repelencia aumentó con la concentración, con un efecto significativamente mayor con concentraciones $\geq 4 \%$. Entre 0,125 y $2 \%$, los aceites fueron neutros o con repelencia débil, y aunque las concentraciones de $2 \%$ de los aceites esenciales obtenidos en las cuatro estaciones causaron IR moderados $(0,51-0,75)$ (Tabla 1$)$, no presentaron diferencias estadísticas con concentraciones menores, con IR cercanos o superiores a 1 (Tabla 3). Estos resultados coinciden con Ortiz et al. (2012) y Norambuena et al. (2016), en que a pesar de que todas las concentraciones de polvo o aceite esencial de hojas de L. philippiana causaron repelencia, las concentraciones mayores tuvieron un índice comparativamente menor lo que implica un mayor efecto repelente; aunque el polvo mostró, en las concentraciones menores, mayor efecto repelente que el aceite esencial. Por su parte, Zapata y Smagghe (2010) evaluaron el aceite esencial de hojas y corteza de L. sempervirens y obtuvieron una fuerte repelencia hacia $T$. castaneum. Los resultados también concuerdan con Cruzat et al. (2009), Nuñez et al. (2010), y Pizarro et al. (2013), quienes concluyeron que si bien todas las concentraciones de polvo de hojas de P. boldus tienen efecto repelente, las mayores causan un efecto mayor. Sin embargo, Pizarro et al. (2013) encontraron que incluso las concentraciones más bajas de polvo $(0,125 ; 0,25$ y $0,5 \% \mathrm{p} / \mathrm{p})$ son capaces de mantener al insecto alejado del grano y evitan posibles reinfestaciones, lo que no ocurrió con el aceite esencial, que para causar un efecto repelente significativo fue necesario utilizarlo al menos al $4 \%$. La diferencia entre esos resultados y los de la presente investigación, es que aquí el valor del IR se analizó estadísticamente, y tratamientos aun inferiores al $4 \%$ a pesar de que presentaron repelencia moderada, no mostraron diferencias significativas con concentraciones que no causaron repelencia, lo cual refrenda la gran variabilidad que tiene el uso de compuestos vegetales para el control de insectos plaga. Así, para una repelencia significativa es recomendable utilizar aceite esencial de $L$. philippiana, $L$. sempervirens o $P$. boldus en concentraciones no inferiores al $4 \%$.

La repelencia obtenida con L. sempervirens y L. philippiana se puede atribuir al alto contenido de safrol y metil-eugenol, y en L. philippiana de safrol potenciado con linalool. Al respecto, Niemeyer y Teillier (2007), Bittner et al. (2008),

Tabla 3. Efecto de la concentración sobre la repelencia (IR) de los aceites esenciales de L. sempervirens, L. philippiana y P. boldus contra adultos de S. zeamais.

Table 3. Effect of the concentration of essential oil of L. sempervirens, L. philippiana, and P. boldus on the repellency $(\mathrm{RI})$ against adults of $S$. zeamais.

\begin{tabular}{cccc}
\hline Especie & L. philippiana & P. boldus & L. sempervirens \\
\hline Concentración (\%) & $----1,----$ & Índice de repelencia (IR) \\
\hline 0,125 & $1,1 \mathrm{a}$ & $1,01 \mathrm{a}$ & $0,96 \mathrm{a}$ \\
0,25 & $1,05 \mathrm{ab}$ & $1,05 \mathrm{a}$ & $0,93 \mathrm{a}$ \\
0,5 & $0,83 \mathrm{ab}$ & $0,82 \mathrm{a}$ & $0,91 \mathrm{ab}$ \\
1,0 & $0,78 \mathrm{ab}$ & $1,10 \mathrm{a}$ & $0,88 \mathrm{ab}$ \\
2,0 & $0,6 \mathrm{bc}$ & $0,72 \mathrm{a}$ & $0,65 \mathrm{bc}$ \\
4,0 & $0,26 \mathrm{c}$ & $0,32 \mathrm{~b}$ & $0,60 \mathrm{c}$ \\
8,0 & $0,23 \mathrm{c}$ & $0,18 \mathrm{~b}$ & $0,38 \mathrm{c}$ \\
$\mathrm{CV}(\%)$ & 18,51 & 15,62 & 11,53 \\
\hline
\end{tabular}

\footnotetext{
* Índice de repelencia $(I R)=2 G /(G+P)$, donde $G=$ porcentaje de insectos de cada tratamiento y $P=$ porcentaje de insectos en el testigo.

* Repellency index $(I R)=2 G /(G+P)$, where $G=$ percentage of insects in each treatment, and $P$ = percentage of insects in the control treatment.

En la columna, los valores con la misma letra minúscula no son significativamente diferentes (Tukey, $\mathrm{p} \leq 0,05$ ). CV: Coeficiente de variación.

Values in a column with the same letter are not significantly different (Tukey, $\mathrm{p} \leq 0.05$ ). CV: Coefficient of variation.
} 
Zapata y Smagghe (2010) y Torres et al. (2015), también señalan que la actividad biológica de L. sempervirens probablemente se debe a que su aceite esencial posee un alto contenido de safrol y metileugenol. Ngoh et al. (1998) indicaron que safrol y en menor medida metileugenol es un repelente poderoso contra ninfas de Periplaneta americana (L.) (Blattidae). Igualmente $\mathrm{Wu}$ et al. (2015) señalan que el aceite esencial de Liriope muscari Bailey, con $42,15 \%$ de metil-eugenol, posee una fuerte repelencia contra Lasioderma serricorne (F.) (Anobiidae), Liposcelis bostrychophila Badonnel (Psocoptera: Liposcelididae) y $T$. castaneum.

En L. philippiana, safrol se podría potenciar con linalool, el que por si solo ha tenido efecto repelente contra Aedes aegypti (L.) (Culicidae) (Müller et al. 2009), S. zeamais (Cansian et al. 2015), Plutella xylostella (L.) (Plutellidae) (Mao-xin et al. 2004) y Lycorma delicatula (White) (Fulgoridae) (Yoon et al. 2011).

En P. boldus, solo predominó el compuesto 1-8 cineol en todas las estaciones, ya que ascaridol se encuentra sólo en el aceite proveniente de hojas colectadas en primavera, por lo que es probable que no sea determinante en su actividad repelente. Sobre 1-8 cineol como compuesto individual, Obeng-Ofori et al. (1997) reportaron repelencia contra S. zeamais y S. granarius (L.), mientras Klocke et al. (1987) encontraron repelencia sobre A. aegypti. Con aceites esenciales de otras plantas que también tienen 1-8 cineol como componente principal se reportó efecto contra S. zeamais (Ukeh et al., 2009), Culex pipiens molestus L. (Culicidae) (Traboulsi et al., 2005), Neotoxoptera formosana (Takahashi) (Aphididae) (Hori y Komatsu, 1997) y T. castaneum (Wang et al., 2006).

Solo L. sempervirens presentó interacción significativa entre la estacionalidad y la concentración, con diferencias estadísticas entre el aceite esencial extraído en verano en las concentraciones de 2 y $4 \%$ con los demás tratamientos y estaciones del año (Tabla 4). Estas dos concentraciones causaron un IR cercano a 0 , lo que indica una repelencia muy alta. La repelencia obtenida al $8 \%$ fue significativamente menor que al 2 y 4 , probablemente debido a un efecto de saturación en las semillas de S. zeamais, lo que coincide con Franzios et al. (1997), quienes indican que la toxicidad de los aceites esenciales no siempre presenta una relación lineal con la concentración de los componentes principales. Los aceites esenciales de $L$. sempervirens extraídos en otoño, primavera e invierno, en estas mismas concentraciones causaron IR cercanos a 1, correspondientes a una repelencia débil. En los tratamientos de 0,125 a 1\%, no hubo diferencia estadística en los IR para las diferentes estaciones del año en que se colectaron hojas, fluctuando entre 0,64 y 1,22, que implica repelencia moderada a nula. $\mathrm{Al} 8 \%$, a pesar que en todas las estaciones de colecta se obtuvo un IR $\leq 0,5$, que corresponde a repelencia alta, no hubo diferencia estadística entre estaciones (Tabla 3). Los resultados concuerdan con Torres et al. (2015), quienes concluyeron que aunque no se encontraron diferencias estadísticas entre tratamientos, las mayores concentraciones de polvo de L. sempervirens presentaron mayor efecto repelente. Torres et al. (2014) tampoco encontraron diferencias al evaluar la repelencia de varias concentraciones de aceite esencial de $L$. sempervirens sobre $S$. zeamais, aunque no se indica la época de muestreo.

Así, los resultados obtenidos con los aceites de las diferentes estaciones corroboran la importancia que tiene registrar la época de muestreo del follaje para obtener un efecto repelente significativo del aceite esencial de $L$. sempervirens. Sin embargo, aunque la tendencia obtenida en los resultados indica claramente que con las concentraciones más altas no importa la temporada de muestreo del follaje, lo ideal es utilizar la menor concentración posible, por lo que la mejor alternativa corresponde a aceite esencial al $2 \%$ y extraído de follaje colectado en verano. Este resultado es relevante para los productores de cereales de la zona sur de Chile, donde $L$. sempervirens es más abundante que $P$. boldus, junto con que el período de cosecha de cereales a fines de verano coincide en que el follaje de $L$. sempervirens produce el aceite esencial con mayor efecto repelente. Además, los costos serían menores, ya que esta recolección no hace necesario almacenar hojas o aceite esencial y por lo mismo no se perderá actividad biológica, ya que según Betancurt et al. (2010) el efecto antixenótico de los aceites esenciales disminuye gradualmente en el tiempo por volatilidad y fotodegradación.

Una explicación a la variación estacional obtenida en $L$. sempervirens se puede inferir al observar el análisis fitoquímico, en el que se encontraron diferencias en las concentraciones y número de los compuestos de acuerdo a la época de muestreo de hojas (Tabla 2). Además de safrol y metil-eugenol, que ocurren en altas concentraciones, otros dos compuestos que presentaron diferencias al comparar el aceite proveniente de material obtenido en verano con el resto de las estaciones, fueron isoterpinoleno $(1,46 \%$ en verano, $0,13 \%$ en otoño, $0 \%$ en invierno y $0,1 \%$ en primavera), y (E)-germacreno $\mathrm{D}(2,31 \%$ en verano, $1,01 \%$ en otoño, $0,72 \%$ en invierno y $0,95 \%$ en primavera). Al respecto, Ramírez et al. (2012) encontraron que el aceite esencial 
Tabla 4. Indice de repelencia del aceite esencial de L. sempervirens sobre adultos de S. zeamais. Table 4. Repellency index of the essential oil of L. sempervirens against adults of $S$. zeamais.

\begin{tabular}{cllcl}
\hline Estación & Otoño & \multicolumn{1}{l}{ Invierno } & \multicolumn{1}{c}{ Primavera } & Verano \\
\hline Concentración & & \multicolumn{1}{l}{ Índice de repelencia (IR)* } & \\
\hline 0,125 & $0,7 \mathrm{Aab}$ & $1,22 \mathrm{Aa}$ & $0,91 \mathrm{Aa}$ & $1,03 \mathrm{Aa}$ \\
0,25 & $0,9 \mathrm{Aa}$ & $1,2 \mathrm{Aa}$ & $0,98 \mathrm{Aa}$ & $0,64 \mathrm{Aab}$ \\
0,5 & $0,9 \mathrm{Aa}$ & $1,03 \mathrm{Aa}$ & $0,95 \mathrm{Aa}$ & $0,74 \mathrm{Aab}$ \\
1,0 & $0,78 \mathrm{Aab}$ & $0,97 \mathrm{Aab}$ & $0,85 \mathrm{Aa}$ & $0,93 \mathrm{Aab}$ \\
2,0 & $0,8 \mathrm{Aab}$ & $0,83 \mathrm{Aab}$ & $0,98 \mathrm{Aa}$ & $0,0 \mathrm{Bc}$ \\
4,0 & $0,76 \mathrm{Aab}$ & $0,71 \mathrm{Aab}$ & $0,85 \mathrm{Aa}$ & $0,07 \mathrm{Bc}$ \\
8,0 & $0,27 \mathrm{Ab}$ & $0,4 \mathrm{Ab}$ & $0,5 \mathrm{Aa}$ & $0,36 \mathrm{Abc}$ \\
\hline
\end{tabular}

\footnotetext{
* Índice de repelencia $(\mathrm{IR})=2 \mathrm{G} /(\mathrm{G}+\mathrm{P})$, donde $\mathrm{G}=$ porcentaje de insectos de cada tratamiento y $\mathrm{P}=$ porcentaje de insectos en el testigo.

${ }^{*}$ Repellency index $(I R)=2 G /(G+P)$, where $G=$ percentage of insects in each treatment, and $P=$ percentage of insects in the control treatment.

En la fila, los valores con la misma letra mayúscula, y en la columna, los valores con la misma letra minúscula, no son significativamente diferentes (Tukey, $\mathrm{p} \leq 0,05$ ). Coeficiente de variación $=11,53 \%$.

Values in a row with the same capital letter, and in a column with the same lowercase letter, are not significantly different (Tukey, $\mathrm{p} \leq 0.05$ ). CV: Coefficient of variation $=11.53 \%$.
}

obtenido desde hojas de Annona cherimola Mill. (Annonaceae), un efectivo insecticida y repelente de larvas y adultos de $A$. aegypti, es rico en sesquiterpenos, en particular germacreno D, su principal constituyente. Nerio et al. (2010) señalan que los compuestos volátiles de los aceites esenciales forman una barrera que disuade a los insectos a entrar en contacto con una superficie tratada, y según Bekele y Hassanali (2001) existe sinergismo entre los componentes de los aceites esenciales, por lo que su uso para control de insectos en forma de aceite esencial sería más eficiente que su principal compuesto aislado. Por lo tanto, safrol sumado a isoterpinoleno y (E)germacreno $\mathrm{D}$ podrían ser los causantes del efecto repelente significativamente mayor observado en el aceite esencial de L. sempervirens cuando su follaje se colecta en verano. Los resultados anteriores permiten concluir que al $2 \%$, el aceite esencial de $L$. sempervirens obtenido en verano presenta efecto repelente muy alto, según la escala de referencia (Tabla 1).

Los bioensayos con aceite esencial de $L$. philippiana y $P$. boldus no presentaron diferencia significativa del efecto repelente entre las diversas estaciones, y todos produjeron IR entre 0,66 y 0,84 , clasificando como con repelencia moderada a débil (Tabla 5). Por consiguiente, al no haber diferencia en la época de extracción del follaje, este se puede obtener en cualquier momento del año. Sin embargo, al analizar las concentraciones de manera independiente, tanto para el aceite esencial de L. philippiana como de $P$. boldus, si hubo una diferencia significativa en la concentración en que se utiliza el aceite esencial, siendo significativamente mayor la repelencia registrada en concentraciones $\geq$ al $2 \%$ que los tratamientos con menor dosis de aceite esencial. Estos resultados proyectan a los aceites esenciales de $L$. sempervirens, L. philippiana y $P$. boldus como repelentes potenciales de insectos en cereales almacenados, aunque es recomendable su posterior validación en esas condiciones.

\section{CONCLUSIONES}

El efecto repelente del aceite esencial de $L$. sempervirens, desde follaje colectado en verano contra adultos de $S$. zeamais fue mayor que en las otras estaciones. Los aceites esenciales de L. philippiana y $P$. boldus no presentaron estacionalidad en su efecto repelente.

\section{AGRADECIMIENTOS}

Proyecto FONDECYT 11110105 de iniciación "Essential oil of Peumus boldus Molina, Laurelia sempervirens (Ruiz et Pau.) Tul and Laureliopsis philippiana Looser as an alternative to synthetic insecticides to the Sitophilus spp. complex and Acanthocelides obtectus Say control in stored seeds". 
Tabla 5. Efecto del muestreo estacional sobre el IR de los aceites esenciales de L. philippiana y $P$. boldus contra adultos de $S$. zeamais.

Table 5. Effect of the sampling season on the RI of the essential oils of L. philippiana and P. boldus against adults of $S$. zeamais.

\begin{tabular}{ccc}
\hline Especie & L. philippiana & $P$. boldus \\
\hline Estación & Índice de repelencia (IR) & Índice de repelencia (IR)* $^{*}$ \\
\hline Otoño & $0,78 \mathrm{a}$ & $0,66 \mathrm{a}$ \\
Invierno & $0,63 \mathrm{a}$ & $0,84 \mathrm{a}$ \\
Primavera & $0,62 \mathrm{a}$ & $0,76 \mathrm{a}$ \\
Verano & $0,73 \mathrm{a}$ & $0,71 \mathrm{a}$ \\
\hline $\mathrm{CV}(\%)$ & 18,51 & 15,62 \\
\hline
\end{tabular}

* Índice de repelencia $(\mathrm{IR})=2 \mathrm{G} /(\mathrm{G}+\mathrm{P})$, donde $\mathrm{G}=$ porcentaje de insectos de cada tratamiento y $\mathrm{P}=$ porcentaje de insectos en el testigo.

* Repellency index $(\mathrm{IR})=2 \mathrm{G} /(\mathrm{G}+\mathrm{P})$, where $\mathrm{G}=$ percentage of insects on each treatments, and $\mathrm{P}$ =percentage insects on the control.

En la columna, los valores con la misma letra minúscula no son significativamente diferentes entre sí (Tukey, $\mathrm{p} \leq 0,05)$. CV: Coeficiente de variación.

Values in a column with the same letter are not significantly different (Tukey, $p \leq 0,05)$. Coefficient of variation.

\section{LITERATURA CITADA}

Bekele, J., and A. Hassanali. 2001. Blend effects in the toxicity of the essential oil constituents of Ocimum kilimandscharicum and Ocimun kenyense (Labiataea) on two post-harvest insect pests. Phytochemistry 57:385-391.

Betancur, J., G. Silva, J.C. Rodríguez, S. Fischer, and N. Zapata. 2010. Insecticidal activity of Peumus boldus Molina essential oil against Sitophilus zeamais Motschulsky. Chilean J. Agric. Res. 70:399-407.

Bittner, M., M. Casanueva, C. Arbert, M. Aguilera, V. Hernández, and J. Becerra. 2008. Effects of essential oils from five plants species against the granary weevil Sitophilus zeamais and Acanthoscelides obtectus. J. Chilean Chem. Soc. 53:1455-1459.

Brown, M., and A. Hebert. 1997. Insect repellents: an overview. J. Am. Acad. Dermatol. 36:243249.

Cansian, R.L., V. Astolfi, R.I. Cardoso, N. Paroul, S.S. Roman, A.A. Mielnoczki-Pereira, G.F. Pauletti and A.J. Mossi. 2015. Insecticidal and repellent activity of the essential oil of Cinnamomum camphora var. Linaoolifera Y. Fujita (Ho-Sho) and Cinnamomum camphora (L.) J. Presl. Var. Hosyo (Hon-Sho) on Sitophilus zeamais Mots. (Coleoptera, Curculionidae). Rev. Bras. Pl. Med. 17:769-773.

Castro, A, D. Henriques, M. Coutinho, N. Terra, and M. de Sena Fernandes. 2011. Tomato plant inheritance of antixenotic resistance to tomato leafminer. Pesq. Agropec. Bras. 46:7480.
Chu, S., Q. Liu, G. Jiang, and Z. Liu. 2011. Chemical composition and insecticidal activity of the essential oil of Amethystea caerulea L. Natural Product Res. 26:1207-1212.

Cruzat, M., G. Silva, H. Serri, y R. Hepp. 2009. Protección de ocho cultivares de trigo con polvo de Peumus boldus Molina contra Sitophilus zeamais Motschulsky. IDESIA (Chile) 37:39-46.

Di Rienzo J.A., F. Casanoves, M.G. Balzarini, L.Gonzalez, M. Tablada, y C.W. Robledo. 2008. InfoStat, versión 2008. Grupo InfoStat, FCA, Universidad Nacional de Córdoba, Argentina.

Evans, NJ. 1985. The effectiveness of various insecticides on some resistant beetle pests of stored products from Uganda. J. Stored Prod. Res. 21:105-109.

Fancelli, M., J. Vendramim, R. Frighetto, e A. Lourenção. 2005. Exsudato glandular de genótipos de tomateiro e desenvolvimento de Bemisia tabaci (Genn.) (Sternorryncha: Aleyrodidae) biótipo B. Neotropical Entomology 34:659-665.

Franzios, G., M. Mirotsou, E. Hatziapostolou, J. Kral, Z.G. Scouras, and P. MavraganiTsipidou. 1997. Insecticidal and genotoxic activities of mint essential oils. J. Agric. \& Food. Chem. 45:2690-2694.

Gómez, K., and A. Gómez. 1984. Statistical procedures for agricultural research. 680 p. 2a. ed. John Wiley \& Sons, New York, USA. 
Hori, M., and H. Komatsu. 1997. Repellency of rosemary oil and its components against the onion aphid, Neotoxoptera formosana (Takahashi) (Homoptera, Aphididae). Appl. Entomol. \& Zool. 32:303-310.

Klocke, J.A., M.V. Darlington, and M.F. Balandrin. 1987. 1,8-Cineole (Eucalyptol), a mosquito feeding and ovipositional repellent from volatile oil of Hemizonia fitchii (Asteraceae). J. Chem. Ecol. 13:2131-2141.

Mao-xin, Z., B. Ling, C. Shao-ying, L. Guangwen, and P. Xiong-fei. 2004. Repellent and oviposition deterrent activities of the essential oil from Mikania micrantha and its compounds on Plutella xylostella. Entomologia Sinica 11:37-45.

Mazzonetto, F., e J. Vendramim. 2003. Efeito de pós de origem vegetal sobre Acanthoscelides obtectus (Say) (Coleóptera: Bruchidae) em feijao armazenado. Neotropical Entomololy 32:145-149.

Müller, G., A. Junnila, J. Butler, V.D. Kravchenko, E.E. Revay, R.W. Weiss, et al. 2009. Efficacy of the botanical repellents geraniol, linalool, and citronella against mosquitoes. J. Vector Ecol. 34:2-8.

Nerio, L, J. Oliver, and E. Stashenko. 2010. Repellent activity of essential oils: A review. Bioresource Technology 101:372-378.

Ngoh, S., L.E.W. Choo, F.Y. Pang, Y. Huang, M.R. Kini, and S.H. Ho. 1998. Insecticidal and repellent properties of nine volatile constituents of essential oils against the American cockroach, Periplaneta americana (L.). Pestic. Sci. 54:261-268.

Niemeyer, H., y S. Teillier. 2007. Aromas de la flora nativa de Chile. Universidad de ChileFundación para la Innovación Agraria (FIA), Santiago, Chile.

Norambuena, C., G. Silva, A. Urbina, I. Figueroa, and J.C. Rodríguez. 2016. Insecticidal activity of Laureliopsis philippiana (Looser) Schoode (Atheropermataceae) essential oil against Sitophilus spp. (Coleoptera: Curculionuidae) Chilean J. Agric. Res. 76:330-336.

Nuñez, P., G. Silva, M. Tapia, R. Hepp, J.C. Rodríguez, y A. Lagunes. 2010. Toxicidad de polvos de follaje de paico (Chenopodium ambrosioides L.) y boldo (Peumus boldus Mol.) solos y en mezcla con carbonato de calcio sobre el gorgojo del maíz (Sitophilus zeamais Motschulsky). Agro-Ciencia/Chil. J. Agric. Anim. Sci 26:71-80.

Obeng-Ofori, D., CH. Reichmuth, and A. Hassanali. 1997. Biological activity of 1,8 cineole, a major component of essential oil of Ocimum kenyense (Ayobangira) against stored product beetles. J. Appl. Ent. 121:237-243.
Ortiz, A., G. Silva, A. Urbina, N. Zapata, J.C. Rodríguez, and A. Lagunes. 2012. Bioactivity of tepa (Laureliopsis philippiana (Looser) Shodde) powder to Sitophilus zeamais Motschulsky control in laboratory. Chilean J. Agric. Res. 72:68-73

Pérez, F., G. Silva, M. Tapia, y R. Hepp. 2007. Variación anual de las propiedades insecticidas de Peumus boldus sobre Sitophilus zeamais. Pesq. Agropec. Bras. 42:633-639

Pérez-Mendoza, J. 1999. Survey of insecticide resistance in mexican populations of maize weevil, Sitophilus zeamais Motschulsky (Coleoptera: Curculionidae). J. Stored. Prod. Res. 35:107-115.

Pimentel, M., L. Faroni, R. Guedes, A. Sousa, and M. Tótola. 2009. Phophine resistance in Brazilian populations of Sitophilus zeamais Motschulsky (Coleoptera: Curculionidae). J. Stored Prod. Res. 45:71-74.

Pizarro, D., G. Silva, M. Tapia, J.C. Rodriguez, A. Urbina, A. Lagunes, et al. 2013. Insecticidal activity of Peumus boldus Molina (Monimiaceae) powder against Sitophilus zeamais Motschulsky (Coleoptera: Curculionidae). Boletín Latinoamericano y del Caribe de Plantas Medicinales y Aromáticas (BLACPMA) 12:420-430.

Ramírez R., F. Mora, J. Avila, L. Rojas, A. Usubillaga, S. Segnini, et al. 2012. Composición química y actividad larvicida del aceite esencial de Annona cherimola Mill. de Los Andes venezolanos contra el mosquito Aedes aegypti (L.). Universidad de Los Andes, Mérida, Venezuela. Revista de la Facultad de Farmacia 53:2-6.

Ribeiro, B., R. Guedes, E. Oliveira, and J. Santos. 2003. Insecticide resistance and synergism in Brazilian populations of Sitophilus zeamais (Coleoptera: Curculiondae). J. Stored Prod. Res. 39:21-31.

Rodríguez, C., G. Silva, y J. Vendramim. 2003. Insecticidas de origen vegetal. p. 87-112. In Silva, G., y R. Hepp. (eds.). Bases para el manejo racional de insecticidas. Universidad de Concepción/ Fundación para la Innovación Agraria, Facultad de Agronomía, Chillán, Chile.

Rodríguez, R., E. Ruiz, y J.P. Elissetche. 2005. Árboles en Chile. Editorial Universidad de Concepción, Concepción, Chile.

Roel, A., e J. Vendramim. 2006. Efeito residual do extrato acetato de etila de Trichilia pallida Swartz (Meliaceae) para lagartas de diferentes idades de Spodoptera frugiperda (J.E. Smith, 1797) (Lepidoptera: Noctuidae). Ciencia Rural 36:1049-1054. 
Tefera, T., S. Mugo, and P. Likhayo. 2011. Effects of insect population density and storage time on grain damage and weight loss in maize due to the maize weevil Sitophilus zeamais and the larger grain borer Prostephanus truncatus. Afric. J. Agric. Res. 6:2249-2254.

Torres, C., G. Silva, M. Tapia, J.C. Rodríguez, A. Urbina, I. Figueroa, et al. 2014. Insecticidal activity of Laurelia sempervirens (Ruiz \& Pav.) Tul. essential oil against Sitophilus zeamais Motschulsky. Chilean J. Agric. Res. 74:421426.

Torres, C., G. Silva, M. Tapia, J.C. Rodríguez, A. Urbina, e I. Figueroa. 2015. Propiedades insecticidas del polvo de Laurelia sempervirens L. para el control de Sitophilus zeamais Motschulsky (Coleoptera: Curculionidae) Boletín Latinoamericano y del Caribe de Plantas Medicinales y Aromáticas (BLACPMA) 14:48-59.

Tretamaterra, P., R. Ianiro, C.G. Athanassiou, and N. Kavallieratos. 2015. Behavioral interactions between Sitophilus zeamais and Tribolium castaneum: the first colonizer matters. J. Pest. Sci. 88:573-581.

Traboulsi, A., S. El-Haj, M. Taobu, N.A. Nader, and A. Mrad. 2005. Repellency and toxicity of aromatic plant extracts against the mosquito Culex pipiens molestus (Diptera:Culicidae). Pest Manag. Sci. 61:597-604.

Ukeh, D., M.A. Birkett, J.A. Pickett, A.S. Bowman, and A.J.M. Luntz. 2009. Repellent activity of alligator pepper, Aframomum melegueta, and ginger, Zingiber officinale, against the maize weevil, Sitophilus zeamais. Phytochemistry 70:751-758.
Vogel, H., I. Razmilic, y U. Doll. 1997. Contenido de aceite esencial y alcaloides en diferentes poblaciones de boldo. Ciencia e Investigación Agraria 24:1-6.

Wang, J., F. Zhu, X.M. Zhou, C.Y. Niu, and C.L. Lei. 2006. Repellent and fumigant activity of essential oil from Artemisia vulgaris to Tribolium castaneum (Herbst) (Coleoptera: Tenebrionidae). J. Stored Prod. Res. 42:339347.

Wu, Y., Z. Wen-Juan, W. Ping-Juan, K. Yang, H. Dong-Ye, W. Jian-Yu, et al. 2015. Contact toxicity and repellency of the essential oil of Liriope muscari (DECN.) Bailey against three insect tobacco storage pests. Molecules 20:1676-1685.

Yoon, C., M. Sang-Rae, J. Jin-Won. S. Youn-Ho, C. Sun-Ran, A. Ki-Su, et al. 2011. Repellency of lavander oil and linalool against spot clothing wax cicada, Lycorma delicatula (Hemiptera: Fulgoridae) and their electrophysiological responses. J. of Asia-Pacific Entomol. 14:411416.

Zapata, N., and G. Smagghe. 2010. Repellency and toxicity of essential oils from the leaves and bark of Laurelia sempervirens and Drimys winteri against Tribolium castaneum. Ind. Crops \& Prod. 32:405-410. 